\title{
The African Turquoise Killifish: A Model for Exploring Vertebrate Aging and Diseases in the Fast Lane
}

\author{
ItAmar Harel ${ }^{1}$ AND AnNe Brunet ${ }^{1,2}$ \\ ${ }^{1}$ Department of Genetics, Stanford University, Stanford, California 94305 \\ ${ }^{2}$ Glenn Laboratories for the Biology of Aging at Stanford, Stanford, California 94305 \\ Correspondence: anne.brunet@stanford.edu
}

\begin{abstract}
Why and how organisms age remains a mystery, and it defines one of the biggest challenges in biology. Aging is also the primary risk factor for many human pathologies, such as cancer, diabetes, cardiovascular diseases, and neurodegenerative diseases. Thus, manipulating the aging rate and potentially postponing the onset of these devastating diseases could have a tremendous impact on human health. Recent studies, relying primarily on nonvertebrate short-lived model systems, have shown the importance of both genetic and environmental factors in modulating the aging rate. However, relatively little is known about aging in vertebrates or what processes may be unique and specific to these complex organisms. Here we discuss how advances in genomics and genome editing have significantly expanded our ability to probe the aging process in a vertebrate system. We highlight recent findings from a naturally short-lived vertebrate, the African turquoise killifish, which provides an attractive platform for exploring mechanisms underlying vertebrate aging and age-related diseases.
\end{abstract}

\section{MODELING AGING IN THE LABORATORY}

Aging research has greatly benefited from investigating short-lived nonvertebrate models (Fig. 1A), specifically yeast (Saccharomyces cerevisiae), worm (Caenorhabditis elegans), and fly (Drosophila melanogaster). These studies have revealed that some pathways, such as the TOR (target of rapamycin) and insulin/IGF (insulinlike growth factor), can regulate the aging process in an evolutionary conserved manner (Kenyon 2010). However, some crucial aspects of human aging and related pathologies cannot be faithfully modeled in nonvertebrates. These aspects include features unique to vertebrate physiology (such as blood, bones, and an adaptive immune system), and "vertebrate-specific" genes (such as $A P O E$ and $p 15 I N K 4 B$ ). On the other hand, the use of classical vertebrate models (namely, the mouse and zebrafish) for this purpose has been drastically limited by their life span, which is relatively long for experimental approaches (up to 3.5 and $5 \mathrm{yr}$, respectively; Tacutu et al. 2013). Insights into the mechanisms of aging have also been provided by the study of exceptionally long-lived vertebrates (Fig. 1A), such as the naked mole rat $(\sim 30 \mathrm{yr})$, the Brandt's bat $(\sim 30 \mathrm{yr})$, and the bow-headed whale $(\sim 200$ yr) (Tacutu et al. 2013). These species represent a unique resource for understanding the mechanisms behind extreme longevity using comparative genomics, proteomics, and cellular approaches (Austad 2010; Gorbunova et al. 2014). However, these long-lived species are less suited for genetic manipulation, life span, or longitudinal studies. What could be the ideal model system for studying vertebrate aging in the laboratory? An exciting approach would be to choose a naturally short-lived vertebrate to systematically probe the principles govern- ing vertebrate aging and age-related diseases (Harel et al. 2015).

\section{THE AFRICAN TURQUOISE KILLIFISH}

First collected in 1968 in the Gonarezhou National Park (Zimbabwe), the turquoise killifish is currently the shortest-lived vertebrate that can be bred in captivity (Genade et al. 2005; Valenzano et al. 2006b; Cellerino et al. 2015; Harel et al. 2015), with a life span of $\sim 4-6$ mo in optimal laboratory conditions (six- to 10-fold shorter-lived than mice and zebrafish, respectively). This fish species has likely evolved a compressed life cycle (30-40 d from an egg to a sexually mature adult) to adapt to its transient habitat-seasonal water ponds present only during a brief rainy season (Fig. 1B). Importantly, despite its short life span, this fish recapitulates stereotypical age-dependent phenotypes and pathologies such as decline in fertility, cognitive functions, muscle mass (sarcopenia), and regenerative capacity, as well as increase in senescence, neurodegeneration, and cancerous lesions (Genade et al. 2005; Valenzano et al. 2006b; Di Cicco et al. 2011; Wendler et al. 2015). Importantly, the turquoise killifish also displays a conserved response to environmental stimuli known to affect aging in other species, such as dietary restriction (Terzibasi et al. 2009), resveratrol (Valenzano et al. 2006b), and temperature (Valenzano et al. 2006a).

Overall, fish offer several advantages as laboratory animals (Schartl 2014). External fertilization provides a clear window for developmental process and increased accessibility for manipulation. Other advantages include a remarkable regenerative capacity and compatibility with high-throughput approaches, specifically genetic 
A

Genetic aging models

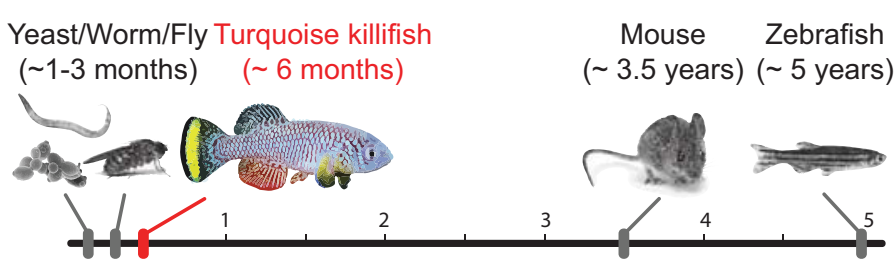

Short-lived models

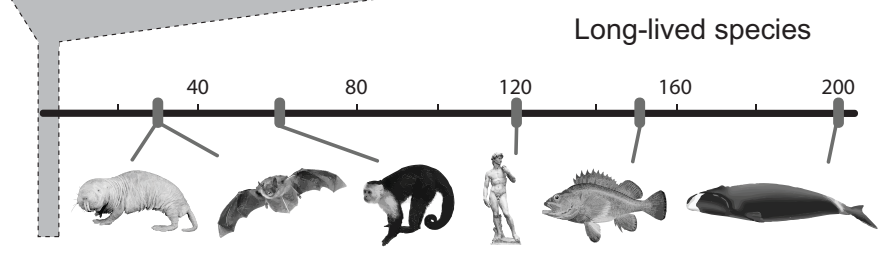

B The African turquoise killifish

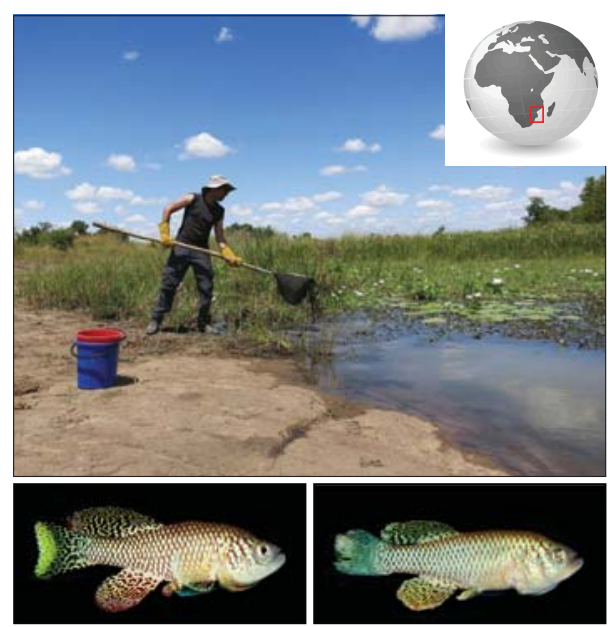

Figure 1. (A) Life span of nonvertebrate and vertebrate genetic model systems used for experimental aging and disease research (top). Life span of long-lived model systems used primarily for cellular and comparative genomic approaches (bottom). (B) The turquoise killifish originates from ephemeral ponds in Zimbabwe and Mozambique (top). Example of a young and old male turquoise killifish (bottom). (Modified from Harel et al. 2015.)

and drug screens. The turquoise killifish has additional advantages, such as an XY-based sexual determination system (Valenzano et al. 2009); this is in contrast to many other fish, including zebrafish. Furthermore, the existence of highly inbred and wild-derived strains (Terzibasi et al. 2008) provides an important advantage for genetic studies and mapping of complex traits such as color and life span (Valenzano et al. 2009; Kirschner et al. 2012). Together, these characteristics make this fish an attractive model organism uniquely fit to study vertebrate aging, physiology, and age-dependent diseases throughout organismal life span. Although many tools have been developed for the turquoise killifish, including genetic linkage maps and Tol2-based transgenesis (Reichwald et al. 2009; Valenzano et al. 2009, 2011; Hartmann and Englert 2012; Kirschner et al. 2012), the lack of a sequenced genome and the ability to manipulate endogenous genes has drastically limited the use of this organism. Thus, for the turquoise killifish to become a widely used vertebrate model, genomic and genome editing approaches had to be generated.

\section{A RAPID GENOME-TO-PHENOTYPE PLATFORM FOR VERTEBRATE AGING}

This challenge was recently addressed by developing a comprehensive genome-to-phenotype platform for this naturally short-lived fish (Fig. 2; Harel et al. 2015). The genomic aspect of this platform included the de novo assembled turquoise killifish genome, RNA sequencing (RNA-seq) in several organs, H3K4me3 (trimethylation of lysine 4 on histone H3) chromatin landscape, and detailed protein homology (Fig. 2). Integrating these genomic data sets made it possible to fully annotate the genome of the turquoise killifish, as well as analyze expression patterns for key aging candidate genes. This genomic platform represents an exciting resource for comparative ge- nomics and evolutionary studies of aging and longevity. Importantly, the annotated genome also set the foundation for developing the second part of this platform - a streamlined genome-editing pipeline (Fig. 2).

The CRISPR/Cas9 (clustered regularly interspaced short palindrome repeats associated Cas9 nuclease) system (Jinek et al. 2012; Cong et al. 2013; Mali et al. 2013), has emerged as an effective approach for introducing targeted mutations in a variety of organisms (for a detailed list, see Hsu et al. 2014), providing a great promise for genetic studies in both model and nonmodel systems. A recent review has defined the main nine "hallmarks of aging" (López-Otín et al. 2013), including telomere attrition, deregulated nutrient sensing, and stem cell exhaustion (Fig. 3A). Several genes within these hallmarks, such as $I G F 1 R$ and $R P S 6 K B 1$, are evolutionary conserved regulators of longevity (Kenyon 2010), whereas others, such as $A P O E$ and TERT, have been implicated in human age-associated diseases (Alzheimer's disease [Rhinn et al. 2013] and dyskeratosis congenita [Armanios 2009], respectively). However, the vast majority of candidate genes and gene variants have not been systematically evaluated in the context of vertebrate aging and age-related diseases. We recently proposed that the turquoise killifish might be uniquely fit for the challenge (Harel et al. 2015). This study used the CRISPR/Cas9 genome-editing system to mutate multiple aging-related genes (Fig. 3A), thus providing a promising approach to rapidly explore the genetics of vertebrate aging and agerelated diseases.

\section{TELOMERASE-DEFICIENT FISH DISPLAY THE FASTEST ONSET OF RELATED HUMAN PATHOLOGIES}

Telomeres, the protective end of chromosomes, shorten with vertebrate aging (Artandi and DePinho 2010), and 


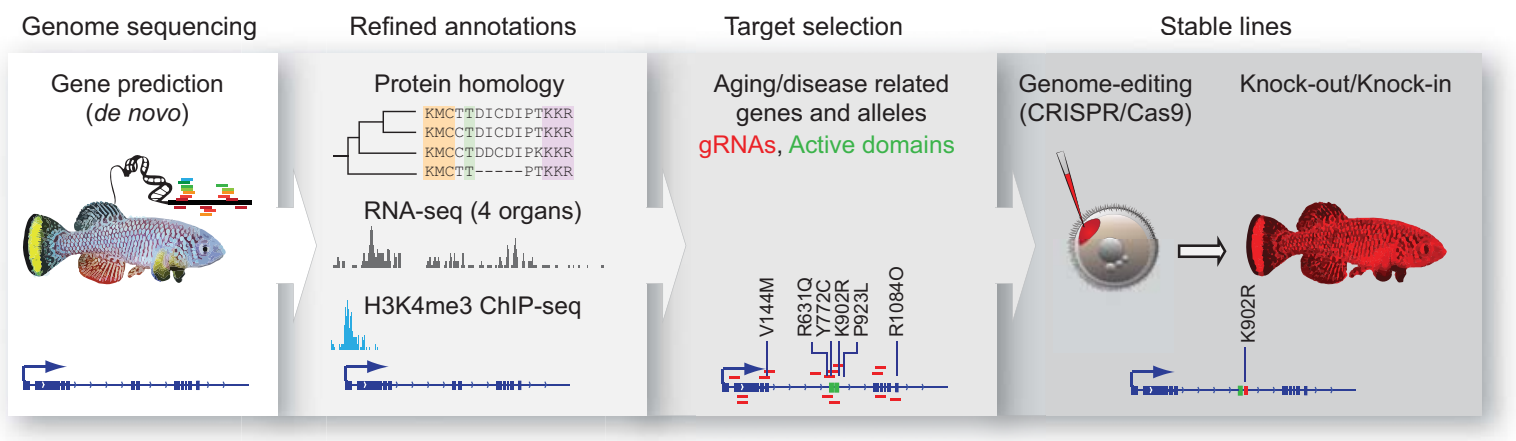

Figure 2. A genome-to-phenotype platform for generating stable mutant fish lines in the turquoise killifish. This pipeline includes genomic tools (de novo assembled turquoise killifish genome, epigenome, and transcriptome) and a CRISPR/Cas9 genome-editing pipeline. (Modified from Harel et al. 2015.)

the rate of telomere attrition is therefore considered to be a good biomarker of biological age (Boonekamp et al. 2013). Mutations in genes required for telomere maintenance have been linked to several diseases, such as dyskeratosis congenita, and patients suffer from a wide range of pathologies affecting mostly highly proliferative tissues. Symptoms include progressive bone marrow failure and pulmonary fibrosis (Armanios and Blackburn 2012), reduced fertility (Bessler et al. 2010), and cancer (Alter et al. 2009), and they resemble some aspects of accelerated aging. The laboratory mouse has exceptionally long telomeres (50-150 kb, Lee et al. 1998), which are $\sim 10$ fold longer then telomere length in humans or the turquoise killifish (Lee et al. 1998; Hartmann et al. 2009; Harel et al. 2015). Consequently, mouse models for telomere-related diseases have to be interbred for about six generations for their telomeres to become critically short and for disease phenotypes to manifest (Lee et al. 1998). Although some alternatives exist, such as the castaneus mouse strain (Hao et al. 2005) and the zebrafish telo- mere-related disease models (Anchelin et al. 2013; Henriques et al. 2013), this has overall greatly limited the ability to model telomere attrition and related pathologies in vivo.

As a proof of principle for rapid genome editing in the turquoise killifish, a loss-of-function allele for the protein component of telomerase $\left(T E R T^{\Delta 8}\right)$ was generated (Fig. 3B), with a total time from injection to a stable line of $\sim 2-3$ mo (Harel et al. 2015). TERT is highly expressed in the vertebrate germline (Bessler et al. 2010) and is essential for maintaining germline "immortality" (Zucchero and Ahmed 2006). Interestingly, TERT mutant fish developed normally, but suffered from a dramatic and progressive decline in fertility (Fig. 3B; Harel et al. 2015). In addition, these mutants displayed defects in other highly proliferative tissues, including the blood and intestine, and showed precancerous changes in the gut, thus recapitulating key aspects of the human syndrome (Harel et al. 2015). Because of cumulative damage in the germline, the offspring of dyskeratosis congenita pa-

A Targeted genes encompassing the "hallmarks of aging"

B A rapid vertebrate model for telomerase-related pathologies
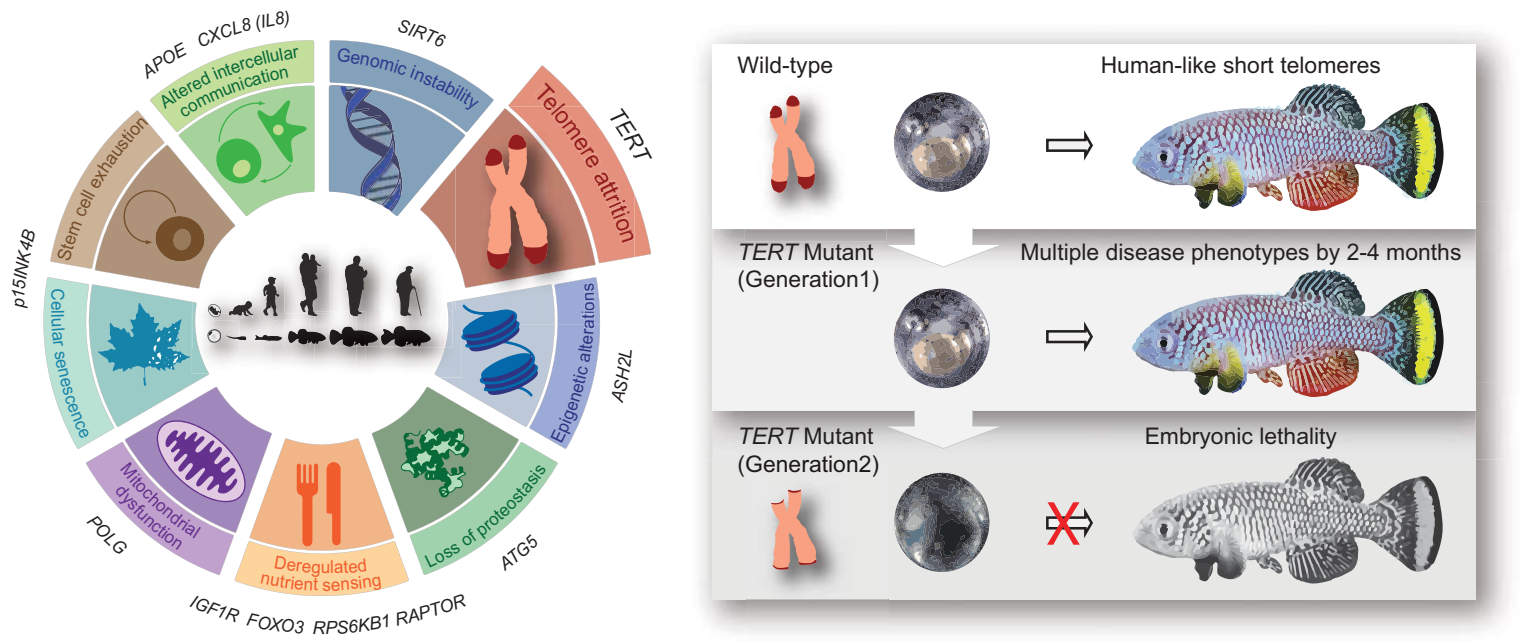

Figure 3. (A) Genes successfully edited in the turquoise killifish, encompassing the hallmarks of human aging. (B) TERT-deficient fish are currently the fastest vertebrate model for modeling related human syndromes. (Modified from Harel et al. 2015.) 
tients can exhibit an earlier onset of phenotypes or an increase in their severity - a phenomenon known as "genetic anticipation." Consistent with this phenomenon, when TERT fish were intercrossed to generate a second generation (G2) of mutants, these G2 mutant embryos showed gross developmental abnormalities and died before hatching - possibly because of critical telomere shortening (Fig. 3B). Interestingly, a large proportion of human diseases, including dyskeratosis congenita, are not caused by large deletions but rather by single-nucleotide mutations that result in nonsynonymous mutations (1000 Genomes Project Consortium et al. 2012). Therefore, a knock-in approach using CRISPR/Cas9 was developed to accurately introduce a mutation in an amino acid of turquoise killifish TERT that is the equivalent of an amino acid in human TERT whose mutation leads to dyskeratosis congenita (Harel et al. 2015).

\section{WHAT IS NEXT?}

The turquoise killifish model for telomere pathologies shows several advantages when compared with other models. Specifically, disease phenotypes in the turquoise killifish are manifested as early as 2 mo, whereas in the zebrafish model they are manifested after 6-8 mo (Anchelin et al. 2013; Henriques et al. 2013) and in the mouse they require several generations of interbreeding (Lee et al. 1998). Therefore, it is currently the fastest vertebrate system to study telomererelated pathologies in vivo. The genetic approaches described above could be further scaled-up to systematically evaluate how specific genetic variants contribute to complex human diseases, traits, and drug responses in the context of aging. The turquoise killifish provides a paradigm for the use of genome sequencing and genome editing as a way to develop a customized model system in fish species. Remarkably, there are more species of fish than the total of all other vertebrate species combined, including birds, reptiles, amphibians, and mammals (Chapman 2009). This incredible diversity has led to extreme evolutionary adaptations, some of which can naturally simulate complex human traits such as life span and longevity-either extremely compressed or exceptionally long (the turquoise killifish and rockfish, respectively).

Aging studies should greatly benefit from studying species beyond conventional model systems. The compressed life cycle of the turquoise killifish, its small size, and relatively cheap husbandry make it uniquely fit for experimental vertebrate aging studies. The recent genometo-phenotype platform developed for this fish (Harel et al. 2015) has transformed it into a promising genetic model system for aging research and has provided the foundations for the development of additional approaches. For example, this fish could be used to better understand how genetic and environmental factors are integrated in the context of aging. Genome-wide genetic screens using RNAi or CRISPR libraries have been successfully used for probing a wide range of cellular net- works, such as responses to drugs and toxins (for review, see Shalem et al. 2015). Using these platforms to explore the hallmarks of aging (such as stress resistance and nutrient sensing) could be complemented by rapid functional in vivo validation using the turquoise killifish model. Similarly, targeted screens could be directly performed in the turquoise killifish, as was recently shown for the zebrafish model (Shah et al. 2015). Combined, these approaches could significantly enhance our ability to explore the phenotypes and diseases of aging, including neurodegeneration and cognitive decline, stem cell exhaustion, and frailty.

\section{ACKNOWLEDGMENTS}

We thank all members of the Brunet lab and are grateful to our coauthors on Harel et al. (2015), from which the figures and related text were adapted. This work was supported by the National Institutes of Health (NIH) DP1AG044848 and the Glenn Laboratories for the Biology of Aging (A.B.) and by the Damon Runyon, Rothschild, and HFSP fellowships (I.H.).

\section{REFERENCES}

Alter BP, Giri N, Savage SA, Rosenberg PS. 2009. Cancer in dyskeratosis congenita. Blood 113: 6549-6557.

Anchelin M, Alcaraz-Pérez F, Martínez CM, Bernabé-García M, Mulero V, Cayuela ML. 2013. Premature aging in telomerasedeficient zebrafish. Dis Model Mech 6: 1101-1112.

Armanios M. 2009. Syndromes of telomere shortening. Annu Rev Genomics Hum Genet 10: 45-61.

Armanios M, Blackburn EH. 2012. The telomere syndromes. Nat Rev Genet 13: 693-704.

Artandi SE, DePinho RA. 2010. Telomeres and telomerase in cancer. Carcinogenesis 31: 9-18.

Austad SN. 2010. Cats, "rats," and bats: The comparative biology of aging in the 21st century. Integr Comp Biol 50: 783-792.

Bessler M, Wilson DB, Mason PJ. 2010. Dyskeratosis congenita. FEBS Lett 584: $3831-3838$.

Boonekamp JJ, Simons MJ, Hemerik L, Verhulst S. 2013. Telomere length behaves as biomarker of somatic redundancy rather than biological age. Aging Cell 12: 330-332.

Cellerino A, Valenzano DR, Reichard M. 2015. From the bush to the bench: The annual Nothobranchius fishes as a new model system in biology. Biol Rev Camb Philos Soc doi: 10.1111 /brv.12183.

Chapman AD. 2009. Numbers of living species in Australia and the world, 2nd ed. Australian Biodiversity Services (ABRS), Toowoomba, Australia. http://155.187.2.69/biodiversity/abrs/ publications/other/species-numbers/2009/pubs/nlsaw-2ndcomplete.pdf.

Cong L, Ran FA, Cox D, Lin S, Barretto R, Habib N, Hsu PD, Wu X, Jiang W, Marraffini LA, et al. 2013. Multiplex genome engineering using CRISPR/Cas systems. Science 339: 819823.

Di Cicco E, Tozzini ET, Rossi G, Cellerino A. 2011. The shortlived annual fish Nothobranchius furzeri shows a typical teleost aging process reinforced by high incidence of age-dependent neoplasias. Exp Gerontol 46: 249-256.

Genade T, Benedetti M, Terzibasi E, Roncaglia P, Valenzano DR, Cattaneo A, Cellerino A. 2005. Annual fishes of the genus Nothobranchius as a model system for aging research. Aging Cell 4: 223-233.

1000 Genomes Project Consortium, Abecasis GR, Auton A, Brooks LD, DePristo MA, Durbin RM, Handsaker RE, Kang HM, Marth GT, McVean GA. 2012. An integrated 
map of genetic variation from 1,092 human genomes. Nature 491: $56-65$.

Gorbunova V, Seluanov A, Zhang Z, Gladyshev VN, Vijg J. 2014. Comparative genetics of longevity and cancer: Insights from long-lived rodents. Nat Rev Genet 15: 531-540.

Hao LY, Armanios M, Strong MA, Karim B, Feldser DM, Huso D, Greider CW. 2005. Short telomeres, even in the presence of telomerase, limit tissue renewal capacity. Cell 123: $1121-$ 1131.

Harel I, Benayoun BA, Machado B, Singh PP, Hu C-K, Pech MF, Valenzano DR, Zhang E, Sharp SC, Artandi SE, Brunet A. 2015. A platform for rapid exploration of aging and diseases in a naturally short-lived vertebrate. Cell 160: 1013-1026.

Hartmann N, Englert C. 2012. A microinjection protocol for the generation of transgenic killifish (species: Nothobranchius furzeri). Dev Dyn 241: 1133-1141.

Hartmann N, Reichwald K, Lechel A, Graf M, Kirschner J, Dorn A, Terzibasi E, Wellner J, Platzer M, Rudolph KL, et al. 2009. Telomeres shorten while Tert expression increases during ageing of the short-lived fish Nothobranchius furzeri. Mech Ageing Dev 130: 290-296.

Henriques CM, Carneiro MC, Tenente IM, Jacinto A, Ferreira MG. 2013. Telomerase is required for zebrafish lifespan. PLoS Genet 9: e1003214.

Hsu PD, Lander ES, Zhang F. 2014. Development and applications of CRISPR-Cas9 for genome engineering. Cell 157: $1262-1278$

Jinek M, Chylinski K, Fonfara I, Hauer M, Doudna JA, Charpentier E. 2012. A programmable dual-RNA-guided DNA endonuclease in adaptive bacterial immunity. Science 337: $816-821$.

Kenyon CJ. 2010. The genetics of ageing. Nature 464: 504512.

Kirschner J, Weber D, Neuschl C, Franke A, Böttger M, Zielke L, Powalsky E, Groth M, Shagin D, Petzold A, et al. 2012. Mapping of quantitative trait loci controlling lifespan in the short-lived fish Nothobranchius furzeri-A new vertebrate model for age research. Aging Cell 11: 252-261.

Lee HW, Blasco MA, Gottlieb GJ, Horner JW II, Greider CW, DePinho RA. 1998. Essential role of mouse telomerase in highly proliferative organs. Nature 392: 569-574.

López-Otín C, Blasco MA, Partridge L, Serrano M, Kroemer G. 2013. The hallmarks of aging. Cell 153: 1194-1217.

Mali P, Yang L, Esvelt KM, Aach J, Guell M, DiCarlo JE, Norville JE, Church GM. 2013. RNA-guided human genome engineering via Cas9. Science 339: 823-826.

Reichwald K, Lauber C, Nanda I, Kirschner J, Hartmann N, Schories S, Gausmann U, Taudien S, Schilhabel MB, Szafranski K, et al. 2009. High tandem repeat content in the genome of the short-lived annual fish Nothobranchius furzeri: A new vertebrate model for aging research. Genome Biol 10: R16.

Rhinn H, Fujita R, Qiang L, Cheng R, Lee JH, Abeliovich A. 2013. Integrative genomics identifies APOE $\epsilon 4$ effectors in Alzheimer's disease. Nature 500: 45-50.

Schartl M. 2014. Beyond the zebrafish: Diverse fish species for modeling human disease. Dis Model Mech 7: 181-192.

Shah AN, Davey CF, Whitebirch AC, Miller AC, Moens CB. 2015. Rapid reverse genetic screening using CRISPR in zebrafish. Nat Methods 12: 535-540.

Shalem O, Sanjana NE, Zhang F. 2015. High-throughput functional genomics using CRISPR-Cas9. Nat Rev Genet 16: 299-311.

Tacutu R, Craig T, Budovsky A, Wuttke D, Lehmann G, Taranukha D, Costa J, Fraifeld VE, de Magalhães JP. 2013. Human Ageing Genomic Resources: Integrated databases and tools for the biology and genetics of ageing. Nucleic Acids Res 41: D1027-D1033.

Terzibasi E, Valenzano DR, Benedetti M, Roncaglia P, Cattaneo A, Domenici L, Cellerino A. 2008. Large differences in aging phenotype between strains of the short-lived annual fish Nothobranchius furzeri. PLoS One 3: e3866.

Terzibasi E, Lefrançois C, Domenici P, Hartmann N, Graf M, Cellerino A. 2009. Effects of dietary restriction on mortality and age-related phenotypes in the short-lived fish Nothobranchius furzeri. Aging Cell 8: 88-99.

Valenzano DR, Terzibasi E, Cattaneo A, Domenici L, Cellerino A. 2006a. Temperature affects longevity and age-related locomotor and cognitive decay in the short-lived fish Nothobranchius FURZERI. Aging Cell 5: 275-278.

Valenzano DR, Terzibasi E, Genade T, Cattaneo A, Domenici L, Cellerino A. 2006b. Resveratrol prolongs lifespan and retards the onset of age-related markers in a short-lived vertebrate. Curr Biol 16: 296-300.

Valenzano DR, Kirschner J, Kamber RA, Zhang E, Weber D, Cellerino A, Englert C, Platzer M, Reichwald K, Brunet A. 2009. Mapping loci associated with tail color and sex determination in the short-lived fish Nothobranchius furzeri. Genetics 183: 1385-1395.

Valenzano DR, Sharp S, Brunet A. 2011. Transposon-mediated transgenesis in the short-lived African killifish Nothobranchius furzeri, a vertebrate model for aging. G3 (Bethesda) 1: $531-538$.

Wendler S, Hartmann N, Hoppe B, Englert C. 2015. Age-dependent decline in fin regenerative capacity in the short-lived fish Nothobranchius furzeri. Aging Cell 14: 857-866.

Zucchero T, Ahmed S. 2006. Genetics of proliferative aging. Exp Gerontol 41: 992-1000. 


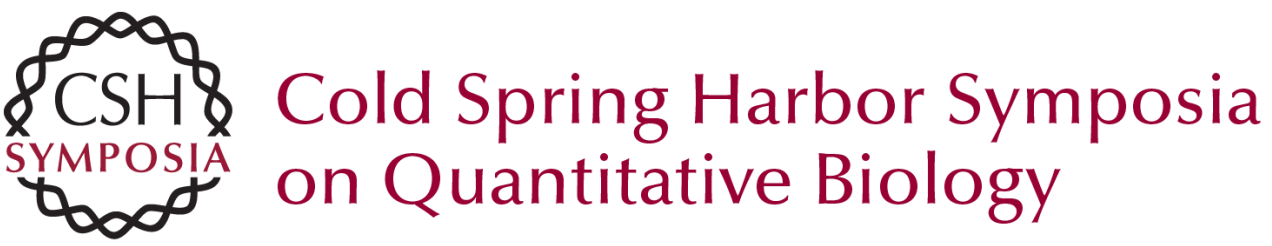

\section{The African Turquoise Killifish: A Model for Exploring Vertebrate Aging and Diseases in the Fast Lane}

Itamar Harel and Anne Brunet

Cold Spring Harb Symp Quant Biol 2015 80: 275-279 originally published online December 7, 2015

Access the most recent version at doi:10.1101/sqb.2015.80.027524

References This article cites 40 articles, 8 of which can be accessed free at: http://symposium.cshlp.org/content/80/275.full.html\#ref-list-1

\section{License}

Email Alerting Receive free email alerts when new articles cite this article - sign up in Service the box at the top right corner of the article or click here. 